\title{
Article
}

\section{Single Phase and Binary Immiscible Fluid Flow in Porous Media Using the Real-coded Lattice Gas}

\author{
Tomonori SAKAI* \\ Centre for Computational Science, Queen Mary College, University of London \\ Mile End Road, London E1 4NS, U.K.* \\ (Received : April 25, 2002)
}

\begin{abstract}
I have developed a model for porous media suitably implemented to the Real-coded Lattice Gas. In order to accurately detect collisions between moving particles and solid matrices, my model investigates the entire trajectories of moving particles. Behaviour of single phase fluids have been validated by the Darcy's Law. For binary immiscible fluids, the simulation results are in good agreement with the generalised Darcy's Law and the conventional LGA studies. Key Words: Flow in porous media/ Darcy's law/ Real-coded Lattice Gas
\end{abstract}

\section{INTRODUCTION}

The analysis of flow in porous media is an outstanding scientific challenge and plays an essential role in technological, environmental, and biological fields such as petroleum recovery, flow of undergroundwater, and the movement of interstitial liquids.

Conventional continuum fluid modelling based on the macroscopic fluid dynamical equations becomes extremely complicated as far as the investigation of multi-phase flow phenomena in complex geometries is concerned. Moreover, the continuous, uniform, and isotropic assumptions on which the macroscopic equations depend are not guaranteed to hold in multi-component fluids where complex and time-evolving interfaces present. On the other hand, fully atomistic approaches which are the antithesis of conventional continuum methods are not viable, since the characteristic size of pores within such media are still usually far larger than molecular scales. Such calculations are out of reach of present day molecular dynamics and will not be accessible within near future.

Mesoscopic models are good candidates for mitigating problems with conventional continuum methods or fully atomistic approaches. Recently, several works have been reported on flow through porous media using Lattice Boltzmann(LB) models where they made considerable success. ${ }^{1)}$ However, in order to analyse flow in porous media where complex phenomena with multiple characteristic length

E-mail : T.Sakai@qmul.ac.uk scales take place simultaneously, bottom-up approaches are more preferable to top-down ones. Though bottom-up LB was recently proposed, its application to flow in porous media has not been attempted to the best of my knowledge.

Dissipative particle dynamics ${ }^{2,3}$ (DPD) and lattice gas Automata $^{4)}$ (LGA) are typical examples of such bottom-up approaches. In LGA, they do not suffer difficulties which arise purely from dealing with porous media. ${ }^{5,6)}$ The necessary procedures to update are fundamentally the same as those with simple geometries, as the location and the movement of every particle are strongly restricted by underlying lattice structure. They only have to judge in every propagation step whether the updated locations of particles are in a region of fluid or obstacle, by just looking at their neighbourmost sites. However, LGA are known to inherently suffer difficulties in accurately reproducing the physics of real fluids and in naturally dealing with energy transport. In addition, the restriction of discrete lattices leads to the rather complicated collision rules, especially in $3 \mathrm{D}$ geometries.

In DPD, particles are allowed to take arbitrary real values both for locations and velocities. Thus especially when one uses a long time step for updates, particles often penetrate through obstacles in a single time step, so that there is no knowing whether they actually collided against obstacles, using only the information about the feature of the updated locations. Accordingly, they make an approximation that a particle does not collide against obstacles when the updated location is within a fluid region, by setting the time step very small, namely, at the expense of the calculation cost. ${ }^{3)}$ 
RLG (the Real-coded Lattice Gas) can be regarded as the continuous version of the conventional discrete LGA, whose prototype was proposed by Malevanets and Kapral as a model for single-phase fluids. ${ }^{7}$ Later it was extended to model binary $^{8)}$ and ternary ${ }^{9,10)}$ fluids as well as modified in fundamental implementations. Due to the release from lattice restrictions in LGA, well known contaminations of physics, such as the loss of Galilean invariance and the velocitydependent pressure, do not show up in the RLG modeled fluids. Also, comparing with LGA, RLG is easier to apply to $3 \mathrm{D}$ geometries. ${ }^{8,10)}$

In a recent study ${ }^{11)} \mathrm{I}$ and coworkers proposed RLG modeled basic washing processes where surfactant solution flow around an oil drop adhered to a wall. There we used a simple geometry, remaining the incorporation of complex geometries as a future work. This paper provides updated development along this line.

Starting from a brief review of RLG in Section 2, Section 3 describes how to construct porous structures and implement interactions between moving particles and solid matrices. I then simulate single phase and binary immiscible fluid flows in two-dimensional porous media in Section 4. Section 5 contains conclusions and comments.

\section{RLG}

The RLG evolves by a particle translation process and a multi-particle collision process. The location of each particle is renewed in the translation process by $\boldsymbol{r}^{\prime}=\boldsymbol{r}+\boldsymbol{v}$ and its velocity in the collision process by $\boldsymbol{v}^{\prime}=\mathrm{V}+\sigma(\boldsymbol{v}-\mathrm{V})$. All particles at the same site, say collision cell, are subject to the same collision, that is, update their velocity simultaneously. $V$ is the mean velocity of the center of mass of all particles within the collision cell and $\sigma$ is a rotation operator whose rotation angle is randomly defined for each cell. Thus the rotation angles for different collision cells are statistically independent. In these equations primes denote values after collisions. This collision operation is equivalent to that in the direct simulation Monte Carlo (DSMC) ${ }^{12)}$ method, except that the pairwise collision in DSMC is replaced by the multi-particle collision. This modification allows RLG to deal with phenomena in mesoscopic and macroscopic scales which are quite costly to handle using DSMC, with rather reasonable computational costs. Mass, momentum and energy are locally conserved during the collision process. Velocity distributions of particles will be Maxwell-Boltzmann when the whole system is relaxed to an equilibrium state. The coarse-grained macroscopic behavior of the RLG has been proved, with the Boltzmann approximation and the Chapman-Enskog expansion, to obey the Navier-Stokes equations. ${ }^{7)}$

The RLG has also been extended into a model for the immiscible binary fluids ${ }^{8)}$ by installing a colour charge for each particle. In particular, one can introduce two kinds of colours, e.g. red and blue, related to the colour charge $C_{n}$ of the $n$th particle,

$$
C_{n}= \begin{cases}+1 & \text { if it is a red particle } \\ -1 & \text { if it is a blue particle }\end{cases}
$$

Similar to the Rothman-Keller two-phase LGA model ${ }^{13)}$, an attractive force between particles of the same colour and a repulsive force between particles of different colours are applied by rotating the colour flux vector

$$
\boldsymbol{Q}(\boldsymbol{r})=\sum_{n}^{N(\boldsymbol{r})} C_{n}\left(\boldsymbol{v}_{n}-\mathrm{V}(\boldsymbol{r})\right)
$$

in reference to the colour field vector

$$
\boldsymbol{F}(\boldsymbol{r})=\sum_{i} \frac{\boldsymbol{r}_{i}-\boldsymbol{r}}{\left|\boldsymbol{r}_{i}-\boldsymbol{r}\right|^{2}} \sum_{n}^{N\left(\boldsymbol{r}_{i}\right)} C_{n}
$$

where $N(\boldsymbol{r})$ is the number of particles in the local cell, $\boldsymbol{v}_{n}$ the velocity of the $n$th particle, $\mathrm{V}(\boldsymbol{r})$ the mean velocity of particles, and $\boldsymbol{r}_{i}$ the $i$ th neighbouring cell. These vectors are defined so as to be analogous to the electrostatic current density and field, when the colour charges are taken to act like electrostatic charges. Ranges of variation for the counter $i$ differ according to the definition of the neighbours. With the 3D Moore neighbours for example, $i$ would be counted from 0 to 26 . To dynamically model the phase separation of the immiscible binary fluid in the simplest way, one may choose a rotation angle for each collision process in a way that the colour flux $\boldsymbol{Q}(\boldsymbol{r})$ could take the same direction as the local colour field vector $\boldsymbol{F}(\boldsymbol{r})$. In other words, the rotation angle is chosen such that the dot product of $\boldsymbol{Q}(\boldsymbol{r})$ and $\boldsymbol{F}(\boldsymbol{r})$ are extremised.

This two-phase RLG has been validated by examining the domain growth in the phase separation process ${ }^{9)}$ and the surface tension which obeys the Laplace's law. ${ }^{8,9)}$

\section{Description and Implementation of Flow in Porous Media}

Two-phase immiscible RLG is useful for generating porous 
structures. Following previous studies ${ }^{5)}$, I simulate off-critical quenches of immiscible two-component fluids, where the phase separation occurs through droplet formation rather than the formation of bicontinuous pattern, after which I defined those domains where the ratio of one component exceeds a specified value as comprising rocks, i.e. solid matrices.

In modelling flow through porous media, some methodologies to drive fluids are indispensable. In this study fluids are driven in the horizontal $(x)$ direction by a gravitational force. That is, a specified value $g$ is added to the $x$ component of velocities of particles at every time step.

One must be careful here to ensure that the solid matrices built do not contain holes or narrow depressions. Otherwise occluded particles, trapped within such pores would gain their kinetic energy every time step by the addition of $g$, without having them dissipated through interactions with particles in other places, see Figure 1. I therefore treated the generated

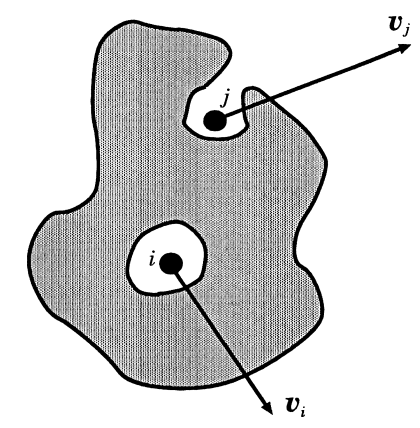

Fig.1 Occluded particles: Some particles can be assigned in a pore completely surrounded by solid matrices at the initial state, like particle $i$. Other particles can be occluded in a depression on the surface of an obstacle, like particle $i$. By adding $g$ to the $x$ components of velocities of these particles at every time step, they will limitlessly gain kinetic energy because their energy can not be dissipated through interactions with other particles.

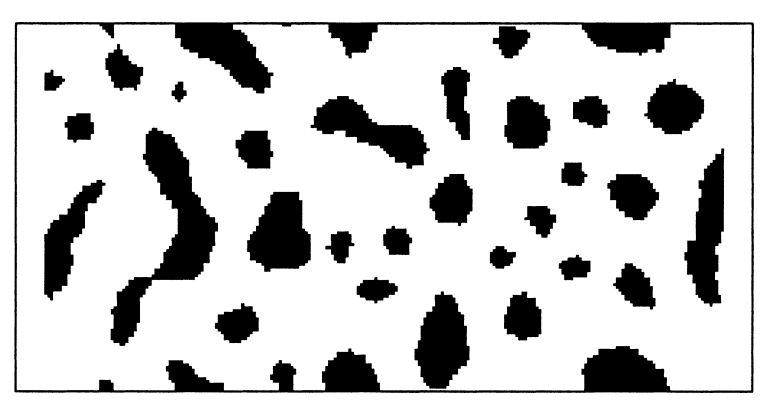

Fig.2 An example of computer-generated two-dimensional porous structure. In order to construct this structure, the off-critical quench of a binary immiscible fluid mixture was simulated, after which domains where the ratio of one component exceeds a specified value were defined as comprisng obstacles. Black: solid matrix; white: pore space. System size is $256 \times 128$, and the porosity is 0.778 . solid matrices with a process for "burying" such pores, if there exsited any. Figure 2 is an example of porous structure finally obtained.

In order to realistically describe multi-phase flows it is necessary to include the wetting properties of the porous media. Within the RLG model, the rock sites are assigned a colour charge $C_{r}$ which may range from $-\rho \leq C_{r} \leq \rho$ where $\rho$ is the number density of particles. In this study I assign colour charges 1.0 and -1.0 to water and oil particles, respectively. $C_{r}$ $=-\rho$ thus represents a completely oil wetting rock.

Within the RLG method, updating the state in porous media simulations requires close attention to the propagation process. Since particles are allowed to assume velocities of arbitrary directions and magnitudes, it frequently happens that a particle penetrates through a rock and reach a fluid area on an other side of the rock. It is thus not enough to know only the information about the starting and ending points of the moving particles, as is done in LGA studies, but their entire trajectories need to be investigated.

Whether a particle hits a solid matrix or not is detected in the following way. First, the cell containing $\boldsymbol{r}^{\prime}=\boldsymbol{r}+\boldsymbol{v}$ is investigated. If this cell is inside a solid matrix the propagation of the particle is rejected and the bounce-back boundary conditions are applied to update. If this cell is within a pore region, a rectangular set of cells where the cells including $\boldsymbol{r}$ and $\boldsymbol{r}^{\prime}$ face each other on the diagonal line is extracted, see Figure 3.

From this set of cells I further extract cells which intercept the trajectory of the particle. In order to do this, every cell $\mathrm{C}_{j}$ in the "box", except those containing $\boldsymbol{r}$ and $\boldsymbol{r}$ ', is investigated

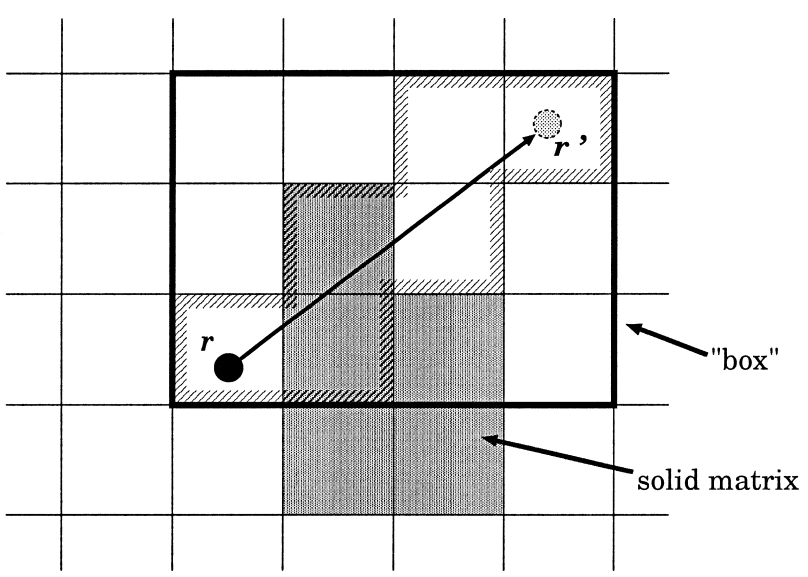

Fig.3 The model to detect whether a particle hits a solid matrix or not. Dark gray area denotes a solid matrix. All the cells in this figure comprise the "box" in which each cell is investigated whether it intercepts the trajectory of the particle. The trajectory passes through dashed cells, which are finally checked whether it is part of a solid matrix or a fluid region. 
by taking the cross product $\boldsymbol{v} \times \mathrm{c}_{j k}$ where $\mathrm{c}_{j k}$ denotes the position vector of four points of a cell $\mathrm{C}_{j}$ and $k=1,2,3$, and 4 . If $\boldsymbol{v} \times \boldsymbol{c}_{\mathrm{jk}}$ 's for all $k$ have the same sign, it means that entire $\mathrm{C}_{j}$ is located on either side of $\boldsymbol{v}$, that is, they do not intercept $\boldsymbol{v}$ and there is no need to check whether the site is inside pore or solid matrices. Otherwise, $\mathrm{C}_{j}$ intercepts $\boldsymbol{v}$ and thus the move is rejected if the site is inside solid matrices, see Figure 3.

This algorithm has significantly increased the number of collisions of particles against solid matrices detected. With the porous medium displayed in Figure 2, it increased by $15 \sim 25 \%$ in runs performed in this study, compared with cases where only the ending points of moving particles are checked.

\section{SIMULATIONS}

In order to validate the model, I performed runs to verify Darcy's Law. Darcy's law relates the flux $\boldsymbol{J}$ of a single phase fluid in porous media to the force applied to the fluid and can be written as

$$
\boldsymbol{J}=\frac{k}{\mu} \boldsymbol{X}
$$

where $k$ is the permeability of the medium, $\mu$ is the dynamic viscosity and $\boldsymbol{X}$ is the applied force due to pressure gradients and gravity. In the porous medium displayed in Figure 2 I forced a single-component fluid of a number density of 10 by adding a varying gravitational driving force to the $x$ component of particle velocities. If the model appropriately

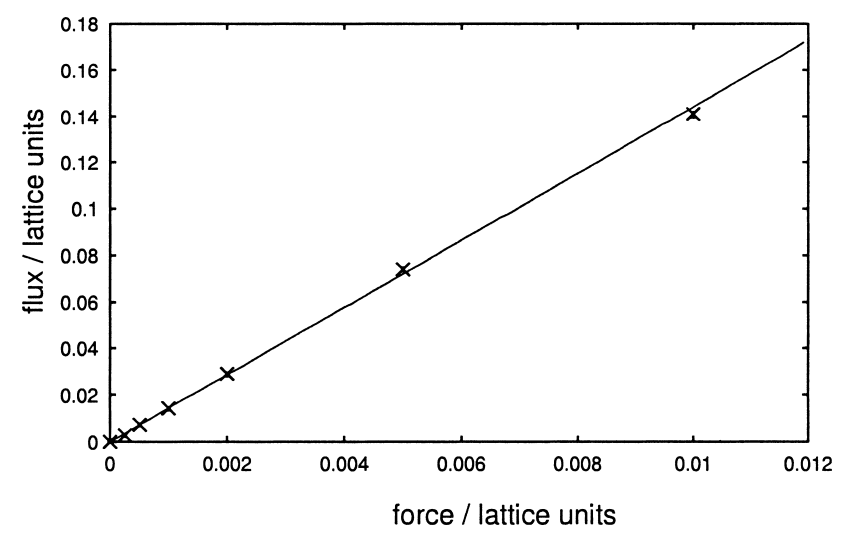

Fig.4 Verification of Darcy's law for a single component fluid in the porous medium displayed in Figure 2. The straight line is a linear fit. The flux is defined as the sum of the $x$ components of particle velocities divided by the number of pore sites. Each data point was obtained by taking the ensemble-average of five runs using different random seeds, where we took an average over 5,000 steps in each. Error bars are so short that they are invisible in the graph. A good linear relationship between the fluid driving force and flux is evident. describes the physics, it is expected from Darcy's Law that the flux of the fluid is proportional to the magnitude of the driving force. Note that $\boldsymbol{J}$ in eqn.(4) is contributed only by the gravitational driving force in the present study, since no pressure gap is applied at right and left hand sides of the system.

Figure 4 shows the results. Each data was obtained by taking the ensemble-average of five different runs, where I took an average over 5,000 steps in each. Linear relations between the magnitude of the forcing and the flux are obvious. Error bars are invisible because they are shorter than the diameters of the points. In a subsequent study ${ }^{14)}$ I and coworkers investigated slopes of the linear fit, by forcing the

(a)

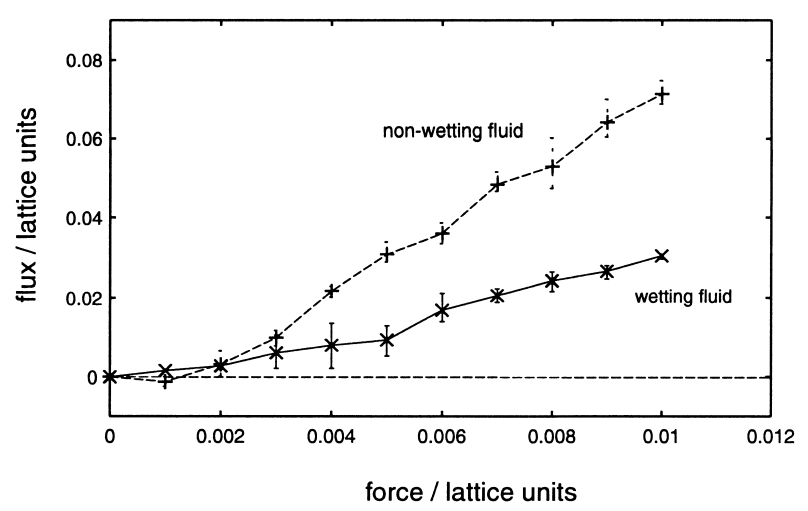

(b)

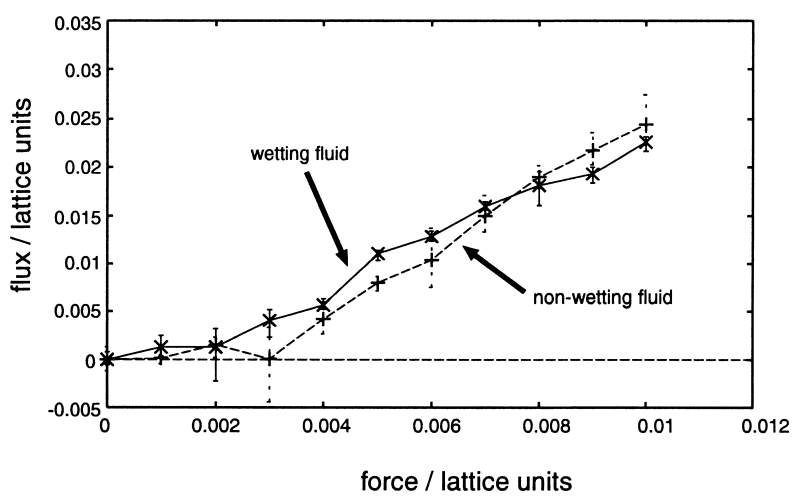

Fig.5 Generalised Darcy behaviour of binary fluids. Mixture of water and oil is forced within a water-wettable porous medium. "Wetting fluid" and "non-wetting fluid" thus correspond to water and oil, respectively. Mean flux of fluids are plotted against the magnitude of the fluid driving force. (a): Responses of forced fluids. i.e. A response of water when water is forced (lower line) and a response of oil when oil is forced (upper line). (b): Responces of unforced fluids. i.e. A response of water when oil is forced and a response of oil when water is forced. I took the ensemble-average of 5 independent runs. In each run, the flux was time-averaged over 5,000 time steps. 
same fluid within three porous structure with roughly the same porosity but different coarsity. The slope of the linear fit, which is proportional to the permeability, was confirmed to be strongly dependent on the chosen porous structure. The validity of the model has thus been confirmed for single phase fluids.

There is no well-established law which governs the binary fluid flow in porous media. One might consider the simplest extension of Darcy's law for two components of the form

$$
\boldsymbol{J}_{i}=k_{i}(S) \frac{k}{\mu_{i}} \boldsymbol{X}_{i}
$$

15) where $\boldsymbol{J}_{i}$ is the flux of the $i$ th species, $k_{i}$ is the relative permeability coefficient (depending on the saturation $S$ ), $\mu_{i}$ is the viscosity, and $\boldsymbol{X}_{i}$ is the body force acting on the $i$ th component. These equations assume that the fluids are uncoupled. However, if one assumes the existence of viscous coupling between the two fluids, the generalised form of Darcy's law becomes

$$
\boldsymbol{J}_{i}=\sum_{j} L_{i j} \boldsymbol{X}_{j}
$$

6) where $i, j=1,2$.

I measured the flux of binary fluids of a number density of 10 in the water-wettable porous medium i.e. where $C_{r}=+\rho=$ +10.0 , shown in Figure 2 with varying magnitude of the forcing. Two types of simulations were performed: one in which only oil was forced, and one in which only water was forced. (The assumption that only one fluid is forced may appear unnatural. Such a state is indeed artificial but is convenient to study the different phenomenological coefficients.) Figure 5 displays the results. In order to take an ensemble-average I performed five independent runs in which data are averaged over 5,000 steps. The results exhibit clear generalised linear Darcy behaviour: there are linear relations between the flux and the forcing for both forced and unforced fluids, in conformity with eqn.(6), when the forcings exceed certain thresholds. The existence of such thresholds is due to the presence of capillary forces, that is, certain forcings are necessary in order that the fluids break their surface tension to flow.

When the forcing level is high enough to cause the flow, there is a significant difference in slopes of water (wetting fluid) and oil (non-wetting fluid) when they are forced; see the upper graph in Figure 5. The steep slope of the oil is caused by lubrication due to the water overlaying the roughness of the solid matrices, while the water adhere to the obstacles, leading to the gentle slope of the water.

One interesting thing here is that the slopes of the unforced fluids are roughly the same, whether the unforced fluid is water or oil; see the lower graph in Figure 5. This indicates that the coupling coefficients between forcings and the fluxes are reciprocal: $L_{12}=L_{21}$ in eqn.(6). Although the theoretical justification remains to be done, this observation is a parallel to the Onsagar's reciprocity ${ }^{16)}$, as seen previously. ${ }^{5,6)}$ These results are therefore encouraging for the further development of the current model.

\section{CONCLUSIONS}

I have reported on the development and implementation of two-dimensional porous media models within the real-coded lattice gas. In order to accurately detect collisions between moving particles and solid matrices, the model investigates the entire trajectories of moving particles.

Behaviour of single phase fluids have been validated by the Darcy's Law. For binary immiscible fluids, the simulation results are in good agreement with the generalised Darcy's Law and the conventional LGA studies.

Extension of this model to deal with ternary amphiphilic fluids is straightforward, for example, by introducing surfactant particles. ${ }^{10)}$ Applications of ternary fluid flows using RLG have so far been limited to those in simple geometries. ${ }^{11)}$ However, it is expected that the applications will include more practical problems relevant to petroleum recoveries, washing processes, groundwater flows and many others where the complex porous structure play a significant role.

\section{Acknowledgements}

I am grateful to Coveney PV and Love PJ for helpful discussions. This work is funded by Ministry of Economy, Trade and Industry of Japan and Daiwa Foundation Europe.

\section{REFERENCES}

1) Martys NS and Chen H, Phys Rev, 53, No.1, 743-750 (1996); Koponen A et. al., Phys Rev Lett, 80, No. 4, 716-719 (1998).

2) Espanol P and Warren P, Europhys Lett, 30, 191 (1995).

3) Koleman JMVA and Hoogerbrugge PJ, Europhys Lett, 21, 369 (1993).

4) Frisch U et. al., Complex Systems, 1, 649 (1994).

5) Malliet J-B and Coveney PV, Int J Mod Phys C, 9, No.8, 14791490 (1998); Malliet J-B and Coveney PV, Phys Rev E 62, $2898(2000)$ 
6) Olson JF and Rothman DH, J Fluid Mech, 341, 343 (1997).

7) Malevanets A and Kapral R, J Chem Phys, 110, 8605 (1999).

8) Hashimoto Y, Chen Y, and Ohashi H, Compu Phys Commu, 129, 56 (2000).

9) Sakai T, Chen Y, and Ohashi H, Phys Rev E, 65, 031503 (2002).

10) Sakai T, Chen $Y$, and Ohashi H, J Collo Surf, 201, 297 (2002).

11) Sakai T, Chen Y, and Ohashi H, Jpn J of Rheo, 29 (2001).
12) Bird GA, Molecular Gas Dynamics and the Direct Simulation of Gas Flows (Clarendon, Oxford) (1994).

13) Rothman DH and Keller J, J Stat Phys, 52, 1119 (1988).

14) Sakai $T$ and Coveney PV, submitted for publication (2002).

15) Gunstensen $\mathrm{AK}$ and Rothman DH, J Geophys Res, 98, 6431 (1993).

16) Onsagar L, Phys Rev, 37, 405 (1931). 